\title{
Multiple Draught Fans Collaborative Control Research of Temperature and Humidity in Drug Finished-Parts Storage
}

\author{
Desheng Liu \\ College of Information and Electronic Technology \\ Jiamusi University \\ Jiamusi, China \\ e-mail: zdhlds@163.com
}

\author{
Zheng Wang \\ College of Information and Electronic Technology \\ Jiamusi University \\ Jiamusi, China \\ e-mail: wang000zheng@163.com
}

\begin{abstract}
With drug finished-parts storage area increases, the control system of fan increases, interactions among multiple fans is inevitable. It is difficult to meet the requirements of the new GMP for finished-parts storage temperature and humidity. Multiple draught fans coordinated fuzzy PID control strategy is proposed in this paper. On the basis of each individual fan control strategy cross coupling method, to reducing the influence on single fan model which the mutual restriction between fan factors. Experimental simulation show that Multiple draught fans coordinated fuzzy PID control strategy can reduce the control time interaction between system for fan by given the influence of different intensity, curtail the volatility of the temperature and humidity range, its control effect is superior to separate control effect of multiple fans.
\end{abstract}

Keywords- Multiple draught fans; Temperature and humidity; Coordinated control; Cross coupling; Fuzzy PID control;

\section{INTRODUCTION}

In recent years, with the surging demand for drugs and the continuous expansion of pharmaceutical company's production, drug finished-parts storage scale is being increased. In order to ensure the environment temperature and humidity in accordance with the storage conditions of the drug, warehouse needs to install more than one air conditioning unit, to ensure that the entire warehouse environment temperature and humidity to achieve the requirement of the drug store. At present, most of the pharmaceutical companies have adopted PID control algorithm to control draught fan [1-2]. With the emergence of a new version of GMP (2011), requirement for pharmaceutical enterprise's warehouse area increasing gradually, stricter and stricter with warehouse's temperature and humidity within the scope of the traditional control scheme and control strategy is very difficult to achieve within the warehouse temperature and humidity control requirements.

In large drug finished-parts storage room, it needs to control more than one fan at the same time, making each fan the temperature and humidity of the region meets the control requirements. The mutual influence among fans causes a lot of interference to moderating effects. The end of each sensor is affected by the cross of the multiple fans at the same time, leading to the asymmetry of temperature and humidity in warehouse. So, some areas can meet the control requirements, while some areas because of the influence of other fan can't achieve the temperature and humidity requirements which depositing to drug the required, leading to drug perishable here and causing direct economic losses to the enterprise [3-4]. To avoid because of the drug in the damage caused by abnormal temperature and humidity to the enterprise, at the same time, as far as possible to improve control precision and the coordinated control of the whole system, this paper put forward the coordinated fuzzy PID control strategy, multiple fans coordinated control model is established, and model for the simulation experiment. The different area of the finished product warehouse temperature and humidity can meet the control requirements, so as to ensure the safety of the finished drug quality.

\section{2. SINGLE FAN CONTROL STRATEGY ANALYSIS}

\section{A. The establishment of a fan model.}

The determination of the transfer function of fan is very important to the whole control system. Assume that the input voltage of the fan is $U$, the output speed is $n$, so the closed-loop transfer function of the fan can be described as follows [5-6]:

$$
W(s)=\frac{N(s)}{U(s)}
$$

Based on the electromagnetic torque formula of fan:

$$
T_{e m}=K_{T} \cdot I_{a}
$$

Where $T_{e m}$ is electromagnetic torque and $K_{T}$ represents the torque coefficient. $I_{a}$ represents current on the armature.

According to the torque balance equation:

$$
T_{e m}=J \frac{d \Omega}{d t}\left(\Omega=\frac{2 \pi n}{60}\right)
$$

Where $J$ is the rotational inertia of the rotor of fan and $\Omega$ represents the armature angular velocity.

Based on mechanical properties of draught fan:

$$
n=\frac{U-I_{a} \cdot R_{a}}{K_{e}}
$$

Where $K_{e}$ is potential coefficient and $R_{a}$ represents the armature resistance. 
From (1), (2) and (3) the $I_{a}, T_{e m}$ and $\Omega$ terms may be eliminated, yielding

$$
K_{e} \cdot n+\frac{2 \pi J R_{a}}{60 K_{T}} \cdot \frac{d n}{d t}=U
$$

Setting the system initial conditions to zero and making Laplace transform of Equation (4), we can rewrite the Equation (4) as the following:

$$
\frac{N(s)}{U(s)}=\frac{1 / K_{e}}{1+\left(J \pi R_{a} / 30 K_{T} \cdot K_{e}\right) s}
$$

Define $\frac{1}{K_{e}}=K, \frac{J \pi R_{a}}{30 K_{T} \cdot K_{e}}=T$, the transfer function model of the fan can be described as follows:

$$
W(s)=\frac{N(s)}{U(s)}=\frac{K}{1+T s}
$$

Define $K=1, T=60$, the transfer function of the fan model in this experiment can be described as follows:

$$
W(s)=\frac{1}{1+60 s}
$$

\section{B. The fuzzy PID control strategy design}

In order to improve the steady state performance of the system, quicken the response speed of each control system, reduce the transition time, the control of each fan adopts fuzzy PID control in this paper [7-8]. The simulation diagram is shown in fig.1. System input deviation and variation rate enter into the fuzzy controller at the same time, in order to make fuzzy processing and fuzzy inference, and then superimpose with the PID control model, and finally make defuzzification by quantitative processing. Making the control signals pass to the fan, and to control the fan. In this paper, the input and output membership function of fuzzy controller adopts triangle. Fuzzy control structure belongs to the adaptive hierarchical fuzzy control. When drug finished-parts storage temperature and humidity have a larger deviation, the system mainly adopts fuzzy control to control fan; when the deviation is small, temperature and humidity system mainly adopts PID control. By setting the deviation threshold, we can switch the two methods.

In this experiment, the deviation and the deviation change rate of the input signal set to 0.01 . The fuzzy controller proportional gain is 0.5 . The integral gain and differential gain is 0.05 . In the PID control model, the proportional gain is 10; integral coefficient is 3 ; differential coefficient is 3.5 .

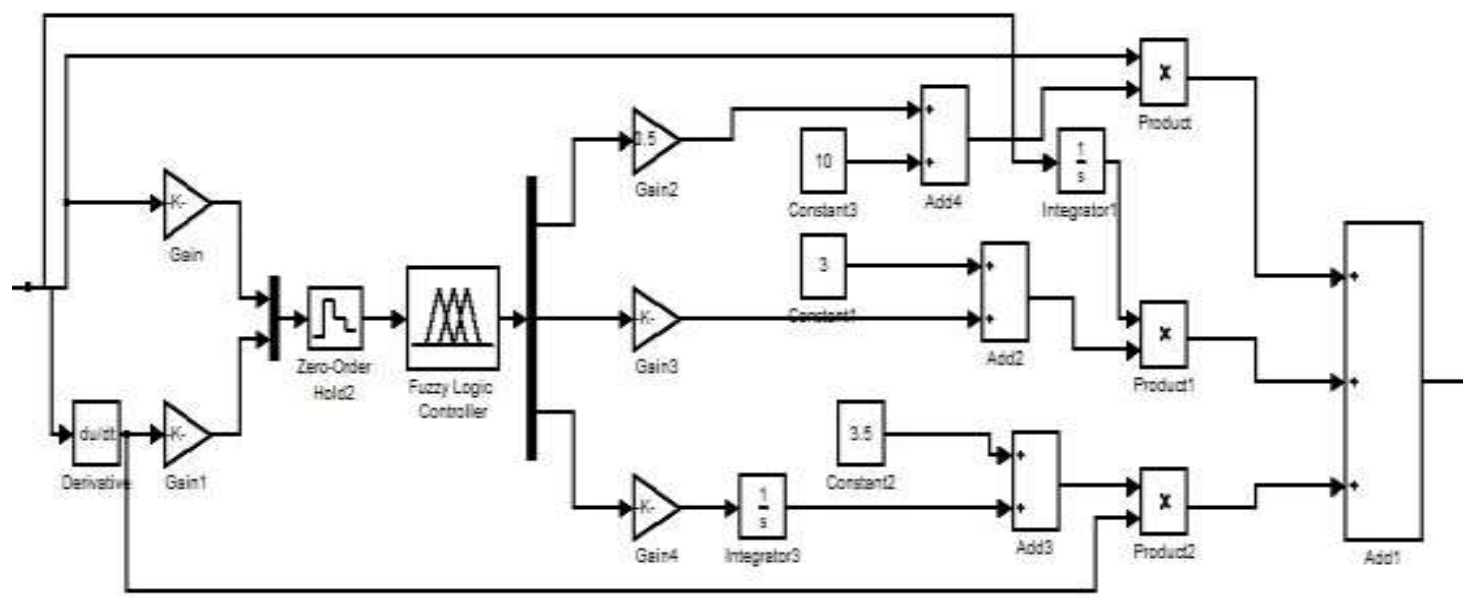

Figure 1. Single fan fuzzy PID control structure

\section{TEMPERATURE AND HUMIDITY CONTROL OF CROSS COUPLING METHOD BASED ON THE MULTIPLE COOPERATING FANS}

In the large drug finished-parts storage temperature and humidity control, not only need to make each fan control effect guaranteed, but also to make the effects of the uniform distribution. It can prevent the mutual interference among the fans, which could not reach the requirements of storing drugs of temperature and humidity in some areas. Therefore, in the process of temperature and humidity control, we can't consider turbine control effect only, but also should take the mutual interference of multiple fans into consideration. So that fans can work collaboratively, and achieve the best control effect. This paper makes the output signal of fan as a feedback signal of control system. And pass to the interference of the fan. The fan gets the output of the other fans, which interfering control effect of the fan. Through its own control system, making the output change timely, so its control effect is stable.

There are two main types of collaborative control technology of the multiple fans control strategy [9-10]: the cross coupling control and the not cross coupling control. In short, the cross coupling control refers to that the dynamic performance of each fan can influence each other in the system, and have visibility to other fans; while the not cross coupling is considered that multiple fan dynamic performance won't influence each other. They have certain independence. The experiment of the control chart is shown in fig.2. Area1 and Area2 have their own 
temperature and humidity control system respectively. But the two control systems have overlapping part of the role of the area. It can make the control action of fan 1 affect sensor2, at the same time, fan 2 control function affects sensor1. Because of their respective sensors being affected, their feedback values are also affected. Thus it makes an impact on their respective control system. Because two fans' system dynamic performances influence each other, this paper selects the cross coupling coordination control strategy. The most important characteristics of the crosscoupling control is that it can compare two fan speed or position signal, so as to get a difference as additional feedback signal; In this way, system is able to reflect any load change, and a fan can obtain good synchronization control precision.

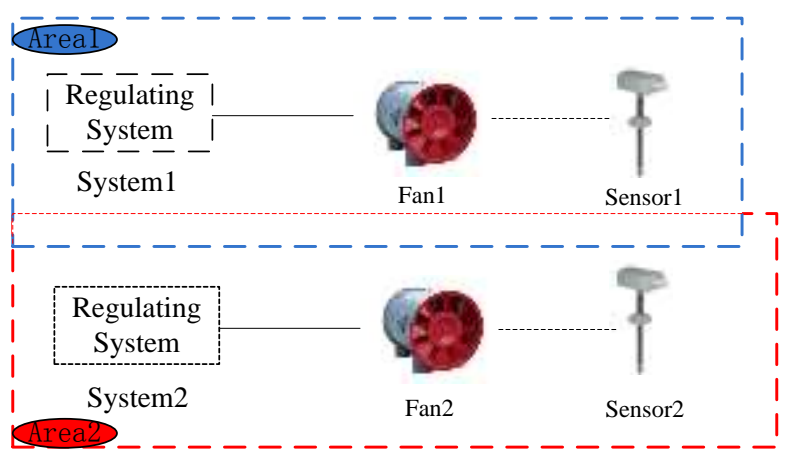

Figure 2. The fan control system schematic diagram

\section{DRUG FINISHED-PARTS STORAGE FAN MORE COLLABORATIVE CONTROL SIMULATION}

The simulation using step signals simulate control system expectations. A single fan control system adopts the fuzzy PID control strategy. In order to simplify the simulation, the experiment chooses two fan control system. The cross coupling coordinated control strategy is used between them, which the difference of output in the two fans feed back to their respective output side. Two fans control system gain setting is $\mathrm{S} 1$ and $\mathrm{S} 2$, in order to simplify the system, the $\mathrm{S} 1$ and $\mathrm{S} 2$ are set to 1 , and join the mutual influence factor of two fan systems as the respective jamming signal.

Drug finished-parts storage multiple draught fans collaborative control simulation model is shown in fig.3. Traditional double fans fuzzy PID control simulation model is shown in fig.4. For the convenience of discussion, we will refer to shown in figure 3 as system1, known shown in figure 4 as system 2 .The the input signal of two systems is step signal, and the amplitude is 1 . The transfer function of fan 1 and fan 2 are the same, the fuzzy PID control strategy parameters are the same as well. K12 is the influence coefficient of fan1 against fan2; K21 is the influence coefficient of fan 2 against fan1. By changing the value of the $\mathrm{K} 12$ and $\mathrm{K} 21$, we can observe variations of the two systems' output under the different impact strength of interference.

\section{Case 1: $K 12=4, K 21=8$}

The simulation output curves of systems 1 and 2 are shown in fig.5. The blue solid line is the output curve of system1 through coordinated fuzzy PID control system; the red dotted line is the system 2 through the fuzzy PID control. Maximum overshoot of output curve of system1 is 0.6 , while the maximum overshoot of system2 is 0.8 . System1 reaching steady state the time required is 73 ; system 2 is 108 .System1's time needed for maximum overshoot is 10 , while system 2 is 17 .

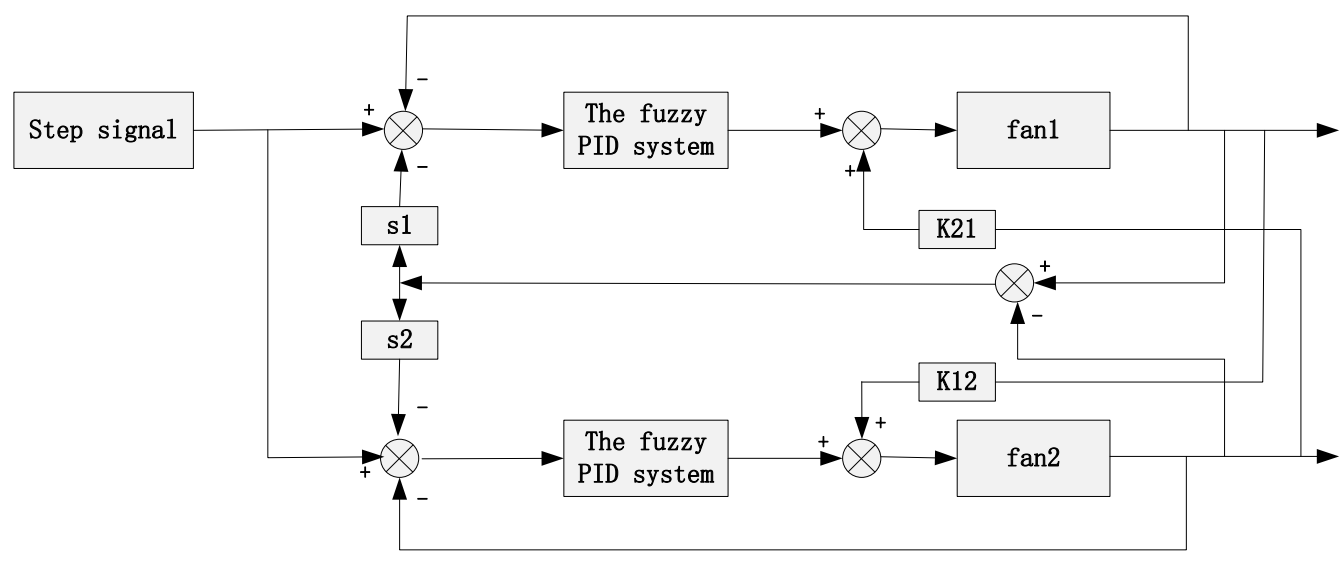

Figure 3. Double fan coordinated control system structure 


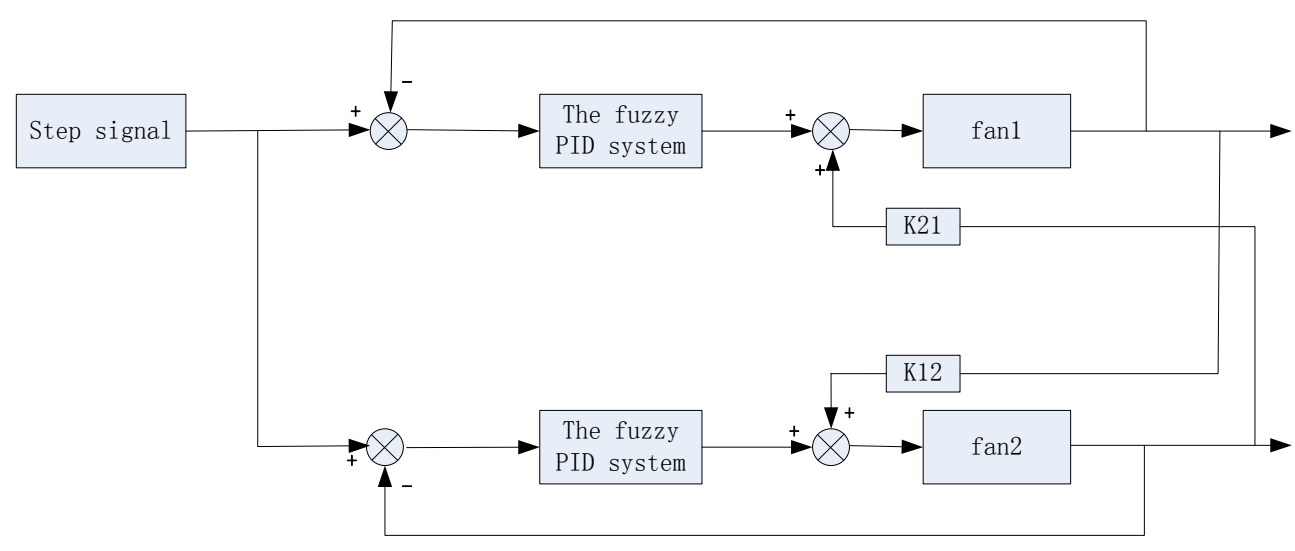

Figure 4. Double fans fuzzy PID system structure

\section{Case 2: $K 12=2, K 21=6$}

The simulation output curves of systems 1 and 2 are shown in fig.6. The blue solid line is the output curve of system1 through coordinated fuzzy PID control system; the red dotted line is the system2 through the fuzzy PID control. Maximum overshoot of output curve of system 1 is 0.46 , while the maximum overshoot of system 2 is 0.65 . System 1 reaching steady state the time required is 65 ; system 2 is 86 . System 1 's time needed for maximum overshoot is 8.6, while system 2 is 15.4 .

Case 3: K12=3, K21=10

The simulation output curves of systems 1 and 2 are shown in fig.6. The blue solid line is the output curve of system1 through coordinated fuzzy PID control system; the red dotted line is the system 2 through the fuzzy PID control. Maximum overshoot of output curve of system 1 is 0.54 , while the maximum overshoot of system 2 is 0.91 . System 1 reaching steady state the time required is 80 ; system 2 is 110. System 1 's time needed for maximum overshoot is 21 , while system 2 is 32 .

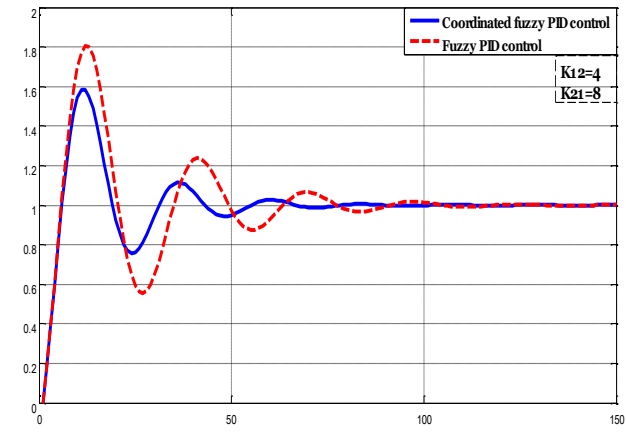

Figure 5. K12 = 4, K21 = 8 parameters output curve

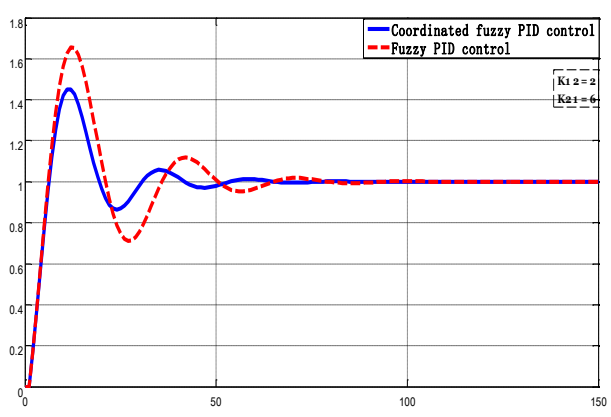

Figure $6 . \mathrm{K} 12=2, \mathrm{~K} 21=6$ parameters output curve

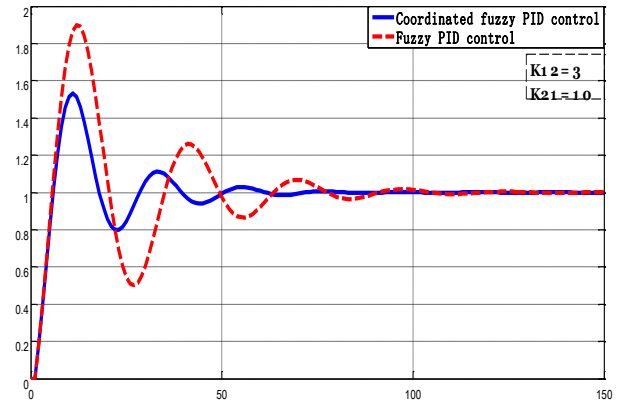

Figure 7. $\mathrm{K} 12=3, \mathrm{~K} 21=10$ parameters output curve

\section{SimUlation RESUlTS AND ANALYSIS}

In Simulation1, the mutual interference coefficient of two fans set to 4 and 8 respectively. Namely, the interference intensity of fan 1 against fan 2 is 4 , and interference intensity of fan 2 against fan 1 is 8 . In this case, output characteristic of fan 1 in system 1 which adopts the coordinated fuzzy PID control strategy is better than the not coordinated control mechanism strategy in the system2; shock strength of curve in system 1 is obviously less than the system2; and the system 1 is earlier than system 2 to reach the preset value. The simulation shows that the control effect of system 1 is better than of system 2 .

Decrease the interfere strength between the fans each other in the two systems at the same time, and reduce the difference value. In this way, we can get the data from simulation2. From the simulation2, we can see that the control effect of system1 is still better than the system2. Compared with the simulation1, the two systems control effect in the simulation1 is better than their respective control effect in the simulation2. And the degree of improvement of the both systems is the same roughly.

In order to see that the strength of the interference between the two fans is how influences on the whole system roundly, we can decrease the K12 in simulation 1 to 3 , and increase $\mathrm{K} 21$ to 10 , and get the simulation experimental results of simulation3. The tendency of the disturbance intensity in simulation 2 and simulation 3 is different. K12 and K21 do not increase or decrease at the same time, but the difference is bigger. From the simulation3, we can see the control effects of variation of the whole system are both worse because of the increase of interference intensity difference. However, the superiority 
of the control effect of system 1 is more obviously than the system 2 .

\section{CONCLUSION}

In recent years, the size of drug finished-parts storage is bigger and bigger, the temperature and humidity control system will be more and more. The mutual influences among the control systems are increasingly apparent, so the effect of temperature and humidity control in warehouse can not meet the drug storage requirements. In order to reduce the mutual interference among the fans, and improve the steady-state performance of the systems, this paper adopts collaborative fuzzy PID control system to control fans.

In this paper, we make a simulation for drug finishedparts storage temperature and humidity control system. By changing the coefficient of mutual interference between fans in the system, we can observe the control effect of the system. Can be seen from the control effect, the system's overshoot amount is decreased obviously, the time needed to stabilize is shortened, and the steady state performance of the overall system is improved. Application of synergy between fuzzy PID controls can diminished the influence significantly which because of interference factors of the fans.

\section{REFERENCES}

[1] Zou Yu, Xiang Fenghong, Wang Jianping, eact. AC Motor Control Strategy Progress [J]. Electric Machines \& Control Application, 2013,03:30-36(In Chinese)

[2] Lin, J, Goo, SY,Chang, JL. Fuzzy coordinator compensation for balancing control of cart-seesaw system. Journal Of Sound And Vibration, 2011:330(26): 6296-6310

[3] Edie, Douglas; Mac skill, Susan; McKellar, Jennifer. Barriers and facilitators to a criminal justice tobacco control coordinator: an innovative approach to supporting smoking cessation among offenders, ADDICTION,2012:107(2) : 26-38

[4] Hu Tao, Tian Chunxiu, Mao Xianqiang. Cooperative Control: Retrospect and Prospect $[\mathrm{J}]$. Environment and Sustainable Development, 2012, 37(1): 25-29(In Chinese)

[5] Wu Dengguo, Li Xiaoming. Modeling and simulation methods for asynchronous motors $[\mathrm{J}]$. Electric Machines \& Control Application,2013,01:1-5. (In Chinese)

[6] $\mathrm{Hu}$ Yusheng, Hu Minqiang. Motor learning [M]. China Electric Power Press. 2009,7.(In Chinese)

[7] Ioannidis, Stratos. Joint production and quality control in production systems with two customer classes and lost sales, IIE Transactions 2013: (Institute of Industrial Engineers) v 45, n 6, p 605-616

[8] Fateh, Mohammad Mehdi; Farahani, Shahab Shahrabi Khatamianfar, Arash. Task Space Control of a Welding Robot Using a Fuzzy Coordinator, International Journal Of Control Automation And Systems, 2010: 8(3) : 574-582

[9] Dong Shiyou, Zhu Xiaping, Long Guoqing. Advances in selforganizing cooperative control $[\mathrm{J}]$. Fire Control \& Command Control, 2013, 38(1): 1-6 (In Chinese)

[10] Olmsted, Russell N.; Kowalski, Christine P.; Krein, Sarah L. Reading habits of infection control coordinators in the United States: Peer-reviewed or non-peer-reviewed evidence? American Journal Of Infection Control 2006: 34(10) : 616-62 